\title{
Microscopic nonlinear quantum theory of absorption of coherent electromagnetic radiation in doped bilayer graphene
}

\author{
A.G. Ghazaryan* and Kh.V. Sedrakian \\ Centre of Strong Fields, Yerevan State University, 1 A. Manukian, Yerevan 0025, Armenia
}

(Dated: July 26, 2019)

\begin{abstract}
The microscopic quantum theory of nonlinear stimulated scattering of chiral particles in doped $A B$ stacked bilayer graphene on Coulomb field of charged impurities in the presence of strong coherent electromagnetic radiation is presented. The Liouville-von Neumann equation for the density matrix is solved analytically. Here the interaction of electrons with the scattering potential is taken into account as a perturbation. The absorption rate of nonlinear inverse-bremsstrahlung for a grand canonical ensemble of fermionic chiral particles is calculated using the obtained solution. The analysis of the obtained rate shows that in the terahertz and near-infrared range of frequencies there is significant absorption of incident radiation via multiphoton stimulated bremsstrahlung mechanism.

PACS numbers: 42.50.Hz, 34.80.Qb, 32.80.Wr, 31.15.-p
\end{abstract}

\section{INTRODUCTION}

Because of the unusual properties of single-layer graphene (SG) [1 6 ] and bilayer graphene (BG) [7-15] the parameter of their interaction with the coherent electromagnetic (EM) radiation is very high in comparison with other systems. The last can allow the fundamental and technological applications of multilayer graphene in nano-opto-electronics, as well as in quantum electrodynamics, low-energy physics, condensed matter physics and at else [16 29]. The high absorption coefficient $\left(\sim 10^{6} \mathrm{~cm}^{-1}\right)$ [30] already cleared that graphene strongly interacts with light. Hence, it is very important that because of the gapless structure of intrinsic SG and $A B$ stacked BG such interaction can be efficiently realized with the terahertz or near-infrared devices as high-power generator and frequency multipliers, as well as a protective material for nanodevices [31], [32]. The absorption of pump wave in nanoscale volumes is a highly desirable property for shielding materials used in nanoelectronics, the aerospace industry, where strict requirements exist such as lightness and smallness or tightness. So, such frequencies are of present interest.

What concerns the absorption of pump wave with the multiphoton stimulated bremsstrahlung (SB), among the wave-induced processes it is an important phenomenon of energy exchange between the charged particles and plane monochromatic wave in plasma-like media [33]. There are many papers relating the electrons elastic scattering on impurity ions in SG, with consideration mainly within the framework of perturbation theory by electrostatic potential 34 42]. Regarding the SB process in graphene at moderate intensities of stimulated radiation, in case of its linear absorption by the charged chiral particles, at the present time, there are extensive investigations carried out in the scope of the linear theory, see, e.g. [43 47]. Multiphoton cross-sections for SB of conduction electrons in intrinsic SG have been obtained in the Born approximation over the scattering potential in the presence of an external EM radiation field in [17], [18].

The interlayer coupling between the two graphene sheets in $\mathrm{AB}$ stacked bilayer graphene strongly change the monolayer's Dirac cone, inducing trigonal warping on the band dispersion and changing the topology of the Fermi surface in the low-energy region. Last changes the transport properties of the BG [7], which studied theoretically within a self-consistent Born approximation in [9]. The rates of elastic and transport scattering on charged impurities in SG and BG are investigated experimentally and comparison with theoretical predictions have been made in [48 53]. The multiphoton rates and total cross-sections of the SB process of conductive electrons on the charged impurity ions in BG had been studied in Born approximation by scattering potential field in [54].

Meanwhile, the interaction of an electron with the EM wave in plasma media at the photon energy $\hbar \omega>T_{e}$ is described by dimensionless intensity parameter of intensity $\chi_{0}=e E_{0} / \omega \sqrt{m_{e} \hbar \omega}$ [16] ( $E_{0}$ is the wave field strength amplitude, $\omega$ is the frequency of the wave, $e$ and $m_{e}$ are the elementary charge and mass, $T_{e}$-plasma temperature). The $\chi_{0}$ is the ratio of the amplitude of the momentum given by the wave field to momentum at the one-photon absorption. The intensity of the wave expressed by the parameter $\chi_{0}$ can be estimated as $I_{\chi_{0}}=\chi_{0}^{2} \times 1.74 \times 10^{12}$ $\mathrm{Wcm}^{-2}(\hbar \omega / \mathrm{eV})^{3}[20]$. Multiphoton effects become essential at $\chi_{0} \sim 1$, which for terahertz photons $\hbar \omega \sim 0.01 \mathrm{eV}$ corresponds to intensity $I_{\chi_{0}} \simeq 10^{6} \mathrm{Wcm}^{-2}$. Meanwhile, in BG for intraband transitions the wave-particle interaction

*Electronic address: amarkos@ysu.am 
at the photon energies $\hbar \omega>\mathcal{E}_{L}[23]$ characterizes by known dimensionless intensity parameter $\chi_{=} \chi_{0}\left(m_{e} \rightarrow m_{*}\right)\left(m_{*}\right.$ is an effective mass of chiral particle, $\mathcal{E}_{L} \simeq 1 \mathrm{meV}$ is the Lifshitz energy). Hence, for the realization of multiphoton $\mathrm{SB}$ in BG, one can expect $\sim 30$ times smaller intensities than for SB in atoms [19], 20], 33, 55, 56].

In the present paper, we have studied the nonlinear absorption coefficient of external EM radiation in doped BG both analytically and numerically. As a mechanism for the real absorption or emission of a plane-monochromatic wave by the charged particles (or plasma-like medium), we have assumed SB process of conductive chiral particles scattering on the charged impurities in doped BG. We developed a microscopic quantum theory of graphene nonlinear interaction with the coherent strong EM radiation of arbitrary. With the help of the solution of Liouville-von Neumann equation for the density matrix, we calculated the nonlinear stimulated scattering of chiral particles in BG on the Coulomb field of impurity ions at the presence of an external pump radiation, taking into account the interaction of charged carriers with the scattering potential in the Born approximation.

In Sec. II the quantum dynamics of SB of conductive chiral particles in BG is presented with analytical results for density matrix and inverse-bremsstrahlung absorption rates. In Sec. III the analytic formulas in case of screened Coulomb field of charged impurities are considered numerically. Conclusions are given in Sec. IV.

\section{THE ABSORPTION COEFFICIENT OF COHERENT EM RADIATION IN DOPED BG}

We will present the quantum theory of multiphoton SB of charged carriers on an arbitrary electrostatic potential of impurity ion in doped bilayer graphene at the presence of the coherent electromagnetic radiation on the base of the density matrix. The pump wave field is considered exactly, while the electrostatic scattering potential of doped ions as a perturbation. To exclude the interband transitions we have considered high Fermi energies and external EM wave is taken to be in the terahertz or near-infrared domain of frequencies. In the presented paper, the influence of multiphoton effects in SB absorption process with an external EM radiation field of moderate intensities is considered. Note, that the first nonrelativistic treatment of SB in the Born approximation has been carried out analytically in the work [33], and then this approach has been extended to the relativistic domain [55].

The pump wave was applied in the perpendicular direction to the BG sheet $(X Y)$ to omit the effect of the magnetic field. Similar calculations for a wave linearly polarized along the $O X$ axis show qualitatively the same picture. The constant phase connected with the position of the wave pulse maximum with respect to the BG plane is set zero. We assume the radiation field to be quazimonochromatic and of the linear polarization with amplitude $E_{0}$ and frequency $\omega=2 \pi / T$ :

$$
\mathbf{E}(t)=\widehat{\mathbf{x}} E_{0} \cos \omega t
$$

The wave vector potential $\mathbf{A}(t)$ will have the form:

$$
\mathbf{A}(t)=-c \int_{0}^{t} \mathbf{E}\left(t^{\prime}\right) d t^{\prime}=-\widehat{\mathbf{x}} \frac{E_{0}}{\omega} \sin \omega t .
$$

The charged impurities are assumed to be at rest and either randomly or nonrandomly distributed in the doped graphene, the arbitrary form electrostatic potential field of which is described by the scalar potential:

$$
\varphi(\mathbf{r})=\sum_{i}^{N_{i}} \phi_{i}\left(\mathbf{r}-\mathbf{R}_{i}\right)
$$

where $\phi_{i}$ is the potential of a single ion placed at the position $\mathbf{R}_{i}$, and $N_{i}$ is the number of impurity ions in the interaction region.

We will consider the quantum kinetic equations for a single chiral particle density matrix for SB process investigation, which can be derived from the second quantized formalism. As a basis for single-particle wave functions we take the approximate solution of the charged chiral particle equation in the strong EM wave field $\mathbf{A}(t)$ we use an even simpler model for BG $A B$ stacked which neglects both the electron-hole asymmetry and the trigonal warping [8], [14], [57, 58]. In accordance with the nonlinear quantum theory of BG in the mentioned case with energy window $0.002 \mathrm{eV} \lesssim \varepsilon \lesssim 0.1$ $\mathrm{eV}$ in the vicinity of Dirac points $K_{\zeta}$ (valley quantum number $\zeta= \pm 1$ ) in the Brillouin zone the fermion particle wave function $\Psi_{\mathbf{p}}(\mathbf{r}, t)$ in the strong EM wave field may be presented in the form:

$$
\Psi_{\mathbf{p}}(\mathbf{r}, t)=F_{\mathbf{p}}(t) e^{\frac{i}{\hbar} \mathbf{p r}} .
$$

Hear $\mathbf{r}=\{x, y\}$ is the 2D-radius vector, parameter $S$ is the quantization area-graphene layer surface area,

$$
F_{\mathbf{p}}(t)=\frac{1}{\sqrt{2 S}}\left(\begin{array}{c}
e^{i 2 \zeta \vartheta} \\
\sigma
\end{array}\right) e^{-i \Omega(\mathbf{p}, t)},
$$




$$
\Omega(\mathbf{p}, t)=\frac{1}{2 m_{*} \hbar} \int\left[\left(p_{x}+\frac{e}{c} A_{x}\right)^{2}+p_{y}^{2}\right] d t,
$$

are the time-dependent spinor wave function $F_{\mathbf{p} \sigma}$ and the phase $\Omega(\mathbf{p}, t)$ (classical action in the field (2)), $\vartheta\left(\mathbf{p}+\frac{e}{c} \mathbf{A}(t)\right)$ is the polar angle in momentum space. BG has a low-energy dispersion which is approximated [12], [58] by massive valence and conduction bands without a gap (in opposite to massless bands in SG). The quasiparticle energy $\mathcal{E}(p)$ is defined by $\mathcal{E}(p)=\sigma\left(p_{x}^{2}+p_{y}^{2}\right) /\left(2 m_{*}\right)$, where $\sigma= \pm 1$ correspond to the conduction/valence bands; $m_{*}=\gamma_{1} /\left(2 \mathrm{v}_{F}^{2}\right)$, $\gamma_{1} \simeq 0.39 \mathrm{eV}$ is the interlayer tunneling amplitude, $\mathrm{v}_{F}$ is the intrinsic SG Fermi velocity. For the actual parameters, the effective mass for BG is $m_{*} \approx(0.033 \div 0.05) m_{e}$. The spin and the valley quantum numbers are conserved. There is no degeneracy upon the valley quantum number $\zeta$, for the issue considered. However, since there are no intervalley transitions, the valley index can be considered as a parameter. The particle states (4) are normalized by the condition

$$
\int \Psi_{\mathbf{p}^{\prime}}^{+}(\mathbf{r}, t) \Psi_{\mathbf{p}}(\mathbf{r}, t) d \mathbf{r}=\frac{(2 \pi \hbar)^{2}}{S} \delta\left(\mathbf{p}-\mathbf{p}^{\prime}\right) .
$$

The second quantized Hamiltonian of the system can be presented in the form:

$$
\mathcal{H}=\mathcal{H}_{0}+\mathcal{H}_{s b}(t)
$$

The first term in Eq. (8) is the Hamiltonian of a single dressed chiral particle

$$
\mathcal{H}_{0}=\int \widehat{\Psi}^{+} \widehat{H}_{0} \widehat{\Psi} d \mathbf{r}
$$

while the second term $\mathcal{H}_{s b}(t)$ is the interaction Hamiltonian describing the SB process in the EM field (2) which can have the form:

$$
\mathcal{H}_{s b}(t)=\frac{1}{c} \int \widehat{j} \varphi(\mathbf{r}) d \mathbf{r}
$$

with the current density operator

$$
\widehat{j}=-e g_{s} g_{\mathrm{v}} \int \widehat{\Psi}^{+}(\mathbf{r}, t) \widehat{\mathbf{v}} \widehat{\Psi}(\mathbf{r}, t) d \mathbf{r}
$$

where $\widehat{\mathbf{v}}=\partial \widehat{H} / \partial \widehat{\mathbf{p}}$ is the velocity operator, $\hat{\mathbf{p}}=\left\{\widehat{p}_{x}, \widehat{p}_{y}\right\}$ is the electron momentum operator, $g_{s}$ and $g_{\mathrm{v}}$ are the spin and valley degeneracy factors, respectively. Here $\widehat{\Psi}$ and $\widehat{H}_{0}$ are the field operator and the Hamiltonian of the dressed single chiral particle in doped BG. In particular, for the effective $2 \times 2$ Hamiltonian [8], 99]:

$$
\widehat{H}_{0}=\frac{1}{2 m_{*}}\left(\begin{array}{cc}
0 & \left(\zeta \widehat{p}_{x}-i \widehat{p}_{y}\right)^{2} \\
\left(\zeta \widehat{p}_{x}+i \widehat{p}_{y}\right)^{2} & 0
\end{array}\right),
$$

the velocity operator in components reads:

$$
\begin{gathered}
\widehat{\mathrm{v}}_{x}=\frac{\zeta}{m_{*}}\left(\begin{array}{cc}
0 & \left(\zeta \widehat{p}_{x}-i \widehat{p}_{y}\right) \\
\left(\zeta \widehat{p}_{x}+i \widehat{p}_{y}\right) & 0
\end{array}\right), \\
\widehat{\mathrm{v}}_{y}=\frac{i}{m_{*}}\left(\begin{array}{cc}
0 & -\left(\zeta \widehat{p}_{x}-i \widehat{p}_{y}\right) \\
\left(\zeta \widehat{p}_{x}+i \widehat{p}_{y}\right) & 0
\end{array}\right) .
\end{gathered}
$$

Note, that the simplified forms for $\widehat{j}$ (11) in doped $A B$ stacked BG in more general cases with taken into account the trigonal warping and induced gap was obtained in [23], [27], [28]. For the following, we will make the Fourier transform of scalar potential in Eq. (10)

$$
\begin{aligned}
\varphi(\mathbf{r}) & =\frac{1}{(2 \pi)^{2}} \int V(\mathbf{q}) e^{-i \mathbf{q} \mathbf{r}} d \mathbf{q} \\
V(\mathbf{q}) & =\int \sum_{i}^{N_{i}} e \varphi_{i}\left(\mathbf{r}-\mathbf{R}_{i}\right) e^{i \mathbf{q} \mathbf{r}} d \mathbf{r}
\end{aligned}
$$


and the interaction Hamiltonian can be expressed in the form:

$$
\mathcal{H}_{s b}(t)=-\frac{g_{s} g_{\mathrm{v}}}{c(2 \pi)^{2}} \iint \widehat{\Psi}^{+} V(\mathbf{q}) e^{-i \mathbf{q} \mathbf{r}} \widehat{\Psi} d \mathbf{q} d \mathbf{r},
$$

where $\hbar \mathbf{q}=\mathbf{p}^{\prime}-\mathbf{p}$ is the recoil momentum.

Let's pass to Furry representation and present the Heisenberg field operator of the chiral particle in the form of an expansion in the quasistationary states (4):

$$
\widehat{\Psi}(\mathbf{r}, t)=\int d \Phi_{\mathbf{p}} \widehat{a}_{\mathbf{p}}(t) \Psi_{\mathbf{p}}(\mathbf{r}, t),
$$

where $\widehat{a}_{\mathbf{p}}(t)$ and $\widehat{a}_{\mathbf{p}}^{+}(t)$ are the annihilation and creation operators, respectively, for a chiral particle with momentum $\mathbf{p}$, associated with positive energy $(\sigma=1)$ solutions satisfy the anticommutation rules at equal times

$$
\begin{gathered}
\left\{\widehat{a}_{\mathbf{p}}^{\dagger}(t), \widehat{a}_{\mathbf{p}^{\prime}}\left(t^{\prime}\right)\right\}_{t=t^{\prime}}=\frac{(2 \pi \hbar)^{2}}{S} \delta\left(\mathbf{p}-\mathbf{p}^{\prime}\right), \\
\left\{\widehat{a}_{\mathbf{p}}^{\dagger}(t), \widehat{a}_{\mathbf{p}^{\prime}}^{\dagger}\left(t^{\prime}\right)\right\}_{t=t^{\prime}}=\left\{\widehat{a}_{\mathbf{p}}(t), \widehat{a}_{\mathbf{p}^{\prime}}\left(t^{\prime}\right)\right\}_{t=t^{\prime}}=0 .
\end{gathered}
$$

where $d \Phi_{\mathbf{p}}=S d^{2} \mathbf{p} d \theta /(2 \pi \hbar)^{2}$ [59]. With the help of Eqs. (11)-(14) and (17) the expectation value of the current for the certain valley $\zeta$ in the case of $A B$ stacked BG can be written as

$$
\widehat{j}=-\frac{e g_{s} g_{v}}{(2 \pi \hbar)^{2} m_{*}} \int \mathbf{p} d \mathbf{p}\left\langle\widehat{a}_{\mathbf{p}}^{+}(t) \widehat{a}_{\mathbf{p}}(t)\right\rangle .
$$

We have excluded the hole operators in Eqs. (17)-(20), since the contribution of electron-holes intermediate states will be negligible for considered EM radiation intensities and Fermi energies. Taking into account anticommutation rules (18), (19) and Eqs. (17), (4)-(6), the second quantized Hamiltonian can be expressed in the form:

$$
H_{0}=\int d \Phi_{\mathbf{p}} \mathcal{E}(\mathbf{p}) \widehat{a}_{\mathbf{p}}^{+} \widehat{a}_{\mathbf{p}}+\mathcal{H}_{s b}(t),
$$

where the first term is the Hamiltonian of the wave dressed two-dimensional chiral particle field, and the second term, which can be expressed by the form:

$$
\mathcal{H}_{s b}(t)=\int d \Phi_{\mathbf{p}} \int d \Phi_{\mathbf{p}^{\prime}} T_{\mathbf{p}^{\prime} \mathbf{p}}(t) \widehat{a}_{\mathbf{p}^{\prime}}^{+} \widehat{a}_{\mathbf{p}}
$$

is the Hamiltonian of interaction describing the SB of the chiral particle with the "quasienergy":

$$
\mathcal{E}(\mathbf{p})=\frac{\omega}{4 \pi m_{*}} \int_{0}^{\frac{2 \pi}{\omega}}\left[\left(p_{x}+\frac{e}{c} A_{x}(t)\right)^{2}+p_{y}^{2}\right] d t
$$

Using the relations (17), (44)-(6) for the impurity potential of the arbitrary form electrostatic potential $V(\mathbf{q})$ we can have the following SB amplitudes:

$$
\begin{gathered}
T_{\mathbf{p}^{\prime} \mathbf{p}}(t)=-\frac{g_{s} g_{v}}{2 S} \int V(\mathbf{q})\left(1+e^{i 2 \zeta\left[\vartheta\left(\mathbf{p}+\frac{e}{c} \mathbf{A}(t)\right)-\vartheta\left(\mathbf{p}^{\prime}+\frac{e}{c} \mathbf{A}(t)\right)\right]}\right) \\
\times e^{-\frac{i}{\hbar} \int_{0}^{\tau}\left[\left(\frac{1}{2 m_{*}}\left(p_{x}^{\prime}+\frac{e}{c} A_{x}\right)^{2}+\frac{p_{y}^{\prime 2}}{2 m_{*}}-\mathcal{E}^{\prime}\right)-\left(\frac{1}{2 m_{*}}\left(p_{x}+\frac{e}{c} A_{x}\right)^{2}+\frac{p_{y}^{2}}{2 m_{*}}-\mathcal{E}\right)\right] d t} .
\end{gathered}
$$

In accordance with Eq. (24) the amplitude $T_{\mathbf{p}^{\prime} \mathbf{p}}(t)$ can be expressed in the following form:

$$
T_{\mathbf{p}^{\prime} \mathbf{p}}(t)=\frac{1}{S} e^{-\frac{i}{\hbar}\left(\mathcal{E}^{\prime}-\mathcal{E}\right) t} B(t)
$$

where the time-depended function

$$
B(t)=-\frac{g_{s} g_{v}}{2} V(\mathbf{q})
$$




$$
\begin{gathered}
\times\left(1+e^{i 2 \zeta\left[\vartheta\left(\mathbf{p}+\frac{e}{c} \mathbf{A}(t)\right)-\vartheta\left(\mathbf{p}^{\prime}+\frac{e}{c} \mathbf{A}(t)\right)\right]}\right) \\
\times e^{-\frac{i}{\hbar} \int_{0}^{\tau}\left[\left(\frac{1}{2 m_{*}}\left(p_{x}^{\prime}+\frac{e}{c} A_{x}\right)^{2}+\frac{p_{y}^{2}}{2 m_{*}}-\mathcal{E}^{\prime}\right)-\left(\frac{1}{2 m_{*}}\left(p_{x}+\frac{e}{c} A_{x}\right)^{2}+\frac{p_{y}^{2}}{2 m_{*}}-\mathcal{E}\right)\right] d t} .
\end{gathered}
$$

Making a Fourier transformation of the function $B(t)$ (26) over $t$, using the known relations

$$
\begin{gathered}
B(t)=\sum_{n=-\infty}^{\infty} e^{-i n \omega t} \widetilde{B}_{\mathbf{p}^{\prime} \mathbf{p}}^{(n)}, \\
\widetilde{B}_{\mathbf{p}^{\prime} \mathbf{p}}^{(n)}=\frac{\omega}{2 \pi} \int_{0}^{2 \pi / \omega} e^{i n \omega t} B(t) d t
\end{gathered}
$$

we can write the SB amplitude $T_{\mathbf{p}^{\prime} \mathbf{p}}(t)(24)$ as

$$
\begin{aligned}
T_{\mathbf{p}^{\prime} \mathbf{p}}(t)= & \frac{1}{S} \sum_{n=-\infty}^{\infty} e^{-i n \omega t} \widetilde{B}_{\mathbf{p}^{\prime} \mathbf{p}}^{(n)} e^{-\frac{i}{\hbar}\left(\mathcal{E}^{\prime}-\mathcal{E}\right) t}= \\
& \frac{1}{S} \sum_{n=-\infty}^{\infty} e^{-i n \omega t} \widetilde{M}_{\mathbf{p}^{\prime} \mathbf{p}}^{(n)}
\end{aligned}
$$

To present the microscopic quantum theory of the multiphoton inverse-bremsstrahlung absorption of coherent EM radiation in doped BG we need to solve the Liouville-von Neumann equation for the density matrix $\widehat{\rho}$ :

$$
\frac{\partial \widehat{\rho}}{\partial t}=\frac{i}{\hbar}\left[\widehat{\rho}, \mathcal{H}_{0}+\mathcal{H}_{s b}(t)\right]
$$

with the initial condition

$$
\widehat{\rho}(0)=\widehat{\rho}_{g}
$$

Before the interaction with EM wave, it is assumed that the system of doped BG quasiparticles was an ideal Fermi gas in equilibrium (thermal and chemical) with a reservoir. Thus the density matrix $\hat{\rho}_{g}$ of the grand canonical ensemble is:

$$
\widehat{\rho}_{g}=\exp \left[\frac{1}{T_{e}}\left(W+\int d \Phi_{\mathbf{p}}(\mu-\mathcal{E}) \widehat{a}_{\mathbf{p}}^{+} \widehat{a}_{\mathbf{p}}\right)\right] .
$$

In Eq. (32) $\mu$ is the chemical potential, $T_{e}$ is the electrons temperature in energy units, $W$ is the grand potential. The initial single-particle density matrix in momentum space will be diagonal, and we will have the fermionic distribution:

$$
\begin{gathered}
\rho\left(\mathbf{p}_{1}, \mathbf{p}_{2}, 0\right)=\operatorname{Tr}\left(\widehat{\rho}_{g} \widehat{a}_{\mathbf{p}_{2}}^{+} \widehat{a}_{\mathbf{p}_{1}}\right)= \\
f\left(P_{1}\right) \frac{(2 \pi \hbar)^{2}}{S} \delta\left(\mathbf{p}_{1}-\mathbf{p}_{2}\right),
\end{gathered}
$$

where

$$
f\left(P_{1}\right)=\frac{1}{\exp \left(\frac{\mathcal{E}_{1}-\mu}{T_{e}}\right)+1} .
$$

Here $\operatorname{Tr}(\widehat{a} \widehat{b} \widehat{c})$ is the trace of a product of the matrices functions $\widehat{a}, \widehat{b}, \widehat{c}$. Within the Born approximation, we consider SB interaction Hamiltonian $\mathcal{H}_{s b}(t)$ as a perturbation. So, we expand the density matrix as

$$
\widehat{\rho}=\widehat{\rho}_{g}+\widehat{\rho}_{1}
$$


taking into account the relations

$$
\begin{gathered}
{\left[\widehat{a}_{\mathbf{p}^{\prime}}^{+} \widehat{a}_{\mathbf{p}}, \widehat{\rho}_{g}\right]=\left(1-e^{\frac{1}{T_{e}}\left(\mathcal{E}^{\prime}-\mathcal{E}\right)}\right) \widehat{\rho}_{g} \widehat{a}_{\mathbf{p}^{\prime}}^{+} \widehat{a}_{\mathbf{p}},} \\
{\left[\widehat{\rho}_{g}, \mathcal{H}_{0}\right]=0,}
\end{gathered}
$$

for $\widehat{\rho}_{1}$ we obtain:

$$
\begin{gathered}
\widehat{\rho}_{1}=-\frac{i}{\hbar} \int_{0}^{t} d t^{\prime} \int d \Phi_{\mathbf{p}} \int d \Phi_{\mathbf{p}^{\prime}} B\left(t^{\prime}\right) \\
\times e^{\frac{i}{\hbar}\left(t^{\prime}-t\right)\left(\mathcal{E}^{\prime}-\mathcal{E}\right)}\left(1-e^{\frac{1}{T_{e}}\left(\mathcal{E}^{\prime}-\mathcal{E}\right)}\right) \widehat{\rho}_{g} \widehat{a}_{\mathbf{p}^{\prime}}^{+} \widehat{a}_{\mathbf{p}} .
\end{gathered}
$$

The energy absorption rate of electrons due to inverse bremsstrahlung can be presented as

$$
\frac{\partial E}{\partial t}=\operatorname{Tr}\left(\widehat{\rho}_{1} \frac{\partial \mathcal{H}_{s b}(t)}{\partial t}\right) .
$$

It is convenient to represent the rate of inverse bremsstrahlung absorption via the mean number of absorbed photons per impurity ion, per unit time:

$$
\frac{d N_{a b s}}{d t}=\frac{1}{\hbar \omega N_{i}} \frac{\partial E}{\partial t}
$$

where $N_{i}$ is the number of impurity ions in the interaction region.

Taking into account the decomposition relation:

$$
\begin{gathered}
\left(1-e^{\frac{1}{T_{e}}\left(\mathcal{E}_{1}-\mathcal{E}_{2}\right)}\right) \operatorname{Tr}\left(\widehat{\rho}_{g} \widehat{a}_{\mathbf{p}_{1}}^{+} \widehat{a}_{\mathbf{p}_{2}} \widehat{a}_{\mathbf{p}_{3}}^{+} \widehat{a}_{\mathbf{p}_{4}}\right)= \\
\left(1-e^{\frac{1}{T_{e}}\left(\mathcal{E}_{1}-\mathcal{E}_{2}\right)}\right) f_{1}\left(1-f_{2}\right),
\end{gathered}
$$

and making the some calculations using the relations (36)-(39), (22), (25), (26) for large $t$ we obtain:

$$
\frac{d N_{a b s}}{d t}=\sum_{n=1}^{\infty} \frac{d N_{a b s}(n)}{d t}
$$

where the partial absorption rates have the following forms:

$$
\begin{gathered}
\frac{d N_{a b s}(n)}{d t}=\frac{4 \pi g_{s} g_{\mathbf{v}} n}{\hbar N_{i} S^{2}} \iint d \Phi_{\mathbf{p}} d \Phi_{\mathbf{p}^{\prime}}|V(\mathbf{q})|^{2}\left|\widetilde{M}_{\mathbf{p}^{\prime} \mathbf{p}}^{(n)}\right|^{2} \\
\times \delta\left(\mathcal{E}^{\prime}-\mathcal{E}+n \hbar \omega\right)\left(1-e^{\frac{1}{T_{e}}\left(\mathcal{E}^{\prime}-\mathcal{E}\right)}\right) \\
\times f\left(\mathcal{E}^{\prime}\right)(1-f(\mathcal{E})),
\end{gathered}
$$

where

$$
\begin{aligned}
& \left|\widetilde{M}_{\mathbf{p}^{\prime} \mathbf{p}}^{(n)}\right|^{2}=\mid \int_{0}^{T} d\left(\frac{t}{T}\right)\left(1+e^{i 2 \zeta\left[\vartheta\left(\mathbf{p}_{0}+\frac{e}{c} \mathbf{A}(t)\right)-\vartheta\left(\mathbf{p}+\frac{e}{c} \mathbf{A}(t)\right)\right]}\right) \exp (i n \omega t) \\
& \times e^{-\left.\frac{i}{\hbar} \int_{0}^{\tau}\left[\left(\frac{1}{2 m_{*}}\left(p_{x}+\frac{e}{c} A_{x}\right)^{2}+\frac{p_{y}^{2}}{2 m_{*}}-\mathcal{E}\right)-\left(\frac{1}{2 m_{*}}\left(p_{0 x}+\frac{e}{c} A_{x}\right)^{2}+\frac{p_{0 y}^{2}}{2 m_{*}}-\mathcal{E}_{0}\right)\right] d t^{\prime}\right|^{2} .} .
\end{aligned}
$$

The obtained expression for the absorption rate is applicable to arbitrary polarization and intensity of the pump wave. It is true for a grand canonical ensemble and positive only. The Dirac $\delta$ function expresses the quadratic dispersion law in the SB process. This parabolic dispersion $\mathcal{E}^{\prime}(p)=\sigma\left(p_{x}^{2}+p_{y}^{2}\right) / 2 m_{*}+n \hbar \omega$ applies only for small values of $p$ satisfying $p / \hbar \ll \gamma_{1} /\left(\hbar \mathrm{v}_{F}\right)$ and for carrier densities (or equivalently, the impurity densities) smaller than $5 \times 10^{12} \mathrm{~cm}^{-2}$. In the opposite limit, $p / \hbar \gg \gamma_{1} /\left(\hbar \mathrm{v}_{F}\right)$ and for carrier densities larger than the last, we get a linear band dispersion, $\varepsilon^{\prime}(p)=\sigma \hbar p \mathrm{v}_{F}+n \hbar \omega$, just as in a case of intrinsic SG [18]. In the following with the help of (42) one can calculate the nonlinear inverse-bremsstrahlung absorption rate for degenerate quantum plasma state - the BG chiral particles with the distribution function given by the Eq. (34). 


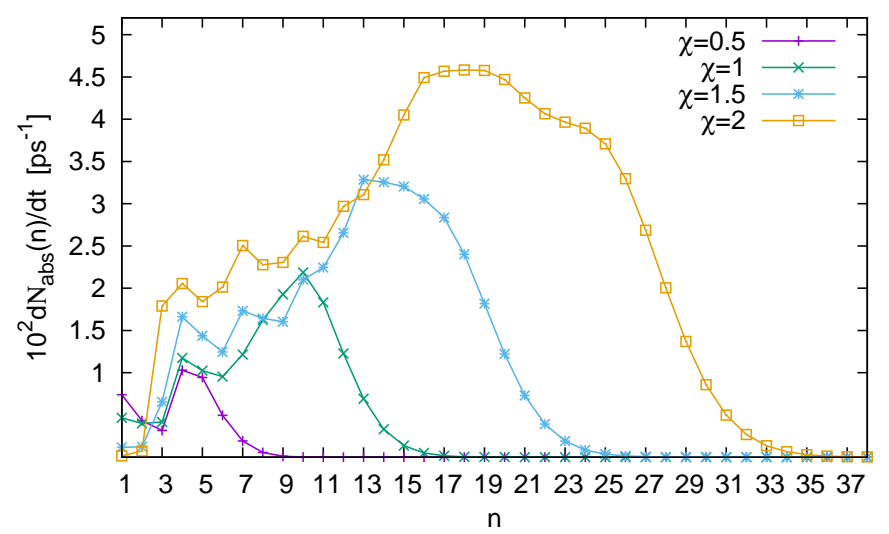

FIG. 1: (Color online) Envelope of partial rate $d N_{a b s}(n) / d t$ of inverse bremsstrahlung absorption vs the mean number of absorbed photons by per ion, per unit time (in $\mathrm{ps}^{-1}$ ) for linear polarization of EM wave in doped graphene is shown for various wave intensities at $\varepsilon \equiv \hbar \omega=0.01 \mathrm{eV}, T_{e}=0.1 \varepsilon_{F}$, and $\varepsilon_{F} \simeq \mu=20 \varepsilon$.

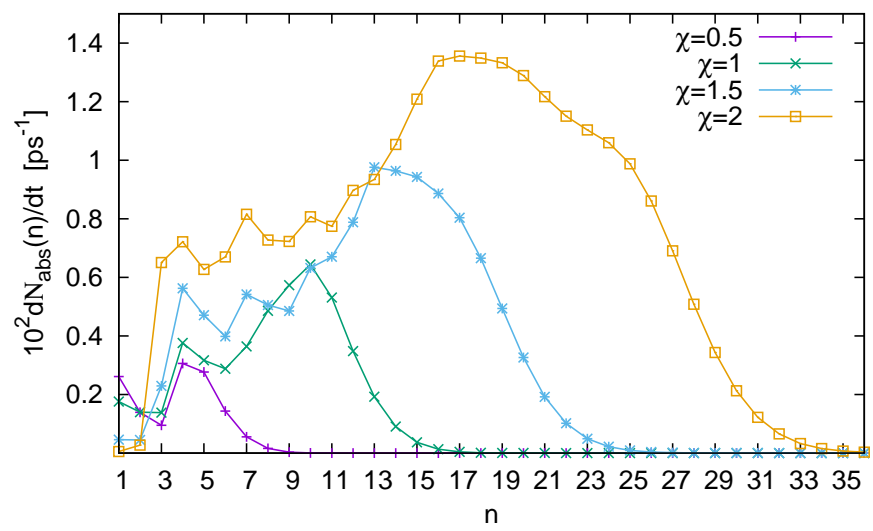

FIG. 2: (Color online) Envelope of partial rate $d N_{a b s}(n) / d t$ of inverse bremsstrahlung absorption vs the mean number of absorbed photons for photon energy $\varepsilon=0.005 \mathrm{eV}$.

\section{NUMERICAL RESULTS FOR SB ABSORPTION COEFFICIENT FOR THE SCREENED COULOMB POTENTIAL OF IMPURITY IONS IN BG}

Now we utilize Eq. (42) in order to obtain the inverse-bremsstrahlung absorption coefficient in particular case of $\mathrm{SB}$ process on a screened Coulomb potential of impurity ions in BG. One we needs to concretize the impurity ionic potential $V(\mathbf{q})$, using an analytic form for Coulomb screening in BG [14], [42, 59, 60]. So, the Fourier transform $V(\mathbf{q})=\int \varphi(\mathbf{r}) e^{-i \mathbf{q r}} d \mathbf{r}$ of a charged impurity center potential can be written as:

$$
|V(\mathbf{q})|^{2}=N_{i} \frac{4 \pi^{2} e^{4}}{\kappa^{2} q^{2} \epsilon^{2}(q)} .
$$

Here the screening term $\epsilon(q)(q=|\mathbf{q}|)$ is the 2D finite temperature static dielectric function in random phase approximation (RPA) appropriate for BG, given by the formula [14], 60] :

$$
\epsilon(q)=1+\frac{q_{s}}{q}\left[g(q)-f(q) \Theta\left(q-2 k_{F}\right)\right] .
$$

Thee $k_{F}=\sqrt{2 m_{*} \varepsilon_{F}} / \hbar$ is $2 \mathrm{D}$ Fermi wave vector in BG case, $q_{s}=k_{T F} / k_{F}=4 m_{*} e^{2} \log 4 /\left(\kappa \hbar^{2}\right)\left(\sim n^{-1 / 2}\right.$ for BG [60]) is the $2 \mathrm{D}$ Thomas-Fermi screening wave vector given by $k_{T F}$ [59] scaled on $k_{F}$; and $\kappa$ is the background lattice dielectric constant of the system. The function $\Theta\left(q-2 k_{F}\right)$ is the step function. The functions $g(q), f(q)$ are defined 


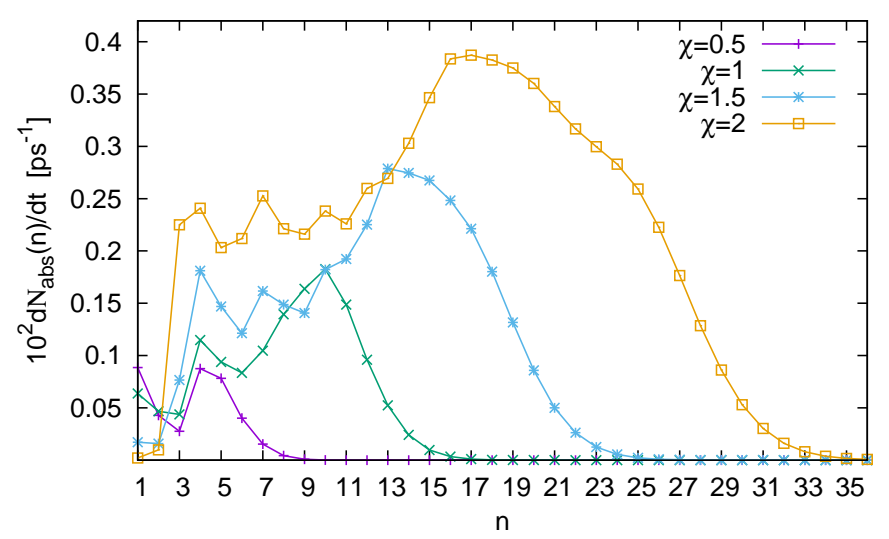

FIG. 3: (Color online) Envelope of partial rate $d N_{a b s}(n) / d t$ of inverse bremsstrahlung absorption vs the mean number of absorbed photons for photon energy $\varepsilon=0.0025 \mathrm{eV}$.

by the formulas [60]:

$$
\begin{gathered}
g(q)=\frac{1}{2} \sqrt{4+\bar{q}^{4}}-\log \left[\frac{1+\sqrt{1+\bar{q}^{4} / 4}}{2}\right], \\
f(q)=\left(1+\frac{\bar{q}^{2}}{2}\right) \sqrt{1-\frac{4}{\bar{q}^{2}}}+\log \left[\frac{\bar{q}-\sqrt{\bar{q}^{2}-4}}{\bar{q}+\sqrt{\bar{q}^{2}-4}}\right],
\end{gathered}
$$

where $\bar{q}=q / k_{F}$. This usual 2D dispersion or the static screening [14] one is the particular case of the wave vector dependent plasmon dispersion and the wave frequency-dependent screening function case [61]. For the last dielectric function and screening show very different behavior than in intrinsic graphene case, and the plasmons creation will be significant.

Taking into account Eqs. (42), (43), and integrating in Eq. (41) over $\mathcal{E}^{\prime}$, we will obtain the following relation for the partial absorption rates $d N_{a b s}(n) / d t$ of SB process:

$$
\begin{aligned}
\frac{d N_{a b s}(n)}{d t} & =\frac{g_{s} g_{\mathrm{v}} n}{\pi \hbar} \frac{\left(\hbar q_{s}\right)^{2}}{16 \log ^{2} 4} \int_{\mathcal{E}+n \hbar \omega} d \mathcal{E} \iint d \theta d \theta^{\prime} \frac{\left|\widetilde{M}_{\mathbf{p}^{\prime} \mathbf{p}}^{(n)}\right|^{2}}{(\hbar q)^{2} \epsilon^{2}(q)} \\
& \times\left(1-e^{-\frac{n \hbar \omega}{T_{e}}}\right) f(\mathcal{E}-n \hbar \omega)(1-f(\mathcal{E})) .
\end{aligned}
$$

For the consideration of numerical results it is convenient to represent the differential cross-sections of SB on the charged impurities in the form of dimensionless quantities. For the dimensionless rates $T d N_{a b s}(n) / d t$ in the field of linearly polarized EM wave with the dimensionless vector potential $\overline{\mathbf{A}}(t)=-\widehat{\mathbf{x}} \chi \sin (2 \pi \tau)$ we have :

$$
\begin{aligned}
T \frac{d N_{a b s}(n)}{d t}= & g_{s} g_{v} n \frac{\left(\overline{\hbar q_{s}}\right)^{2}}{8 \log ^{2} 4} \int_{\overline{\mathcal{E}}+n} d \overline{\mathcal{E}} \iint d \theta_{\mathbf{p}} d \theta_{\mathbf{p}^{\prime}} \frac{\left|\widetilde{M}_{\mathbf{p}^{\prime} \mathbf{p}}^{(n)}\right|^{2}}{|\hbar \overline{\mathbf{q}}|^{2} \epsilon^{2}(|\overline{\mathbf{q}}|)} \\
& \times\left(1-e^{-\frac{n}{T_{e}}}\right) f(\overline{\mathcal{E}}-n)(1-f(\overline{\mathcal{E}})),
\end{aligned}
$$

where

$$
\left|\bar{M}_{\mathbf{p}^{\prime} \mathbf{p}}^{(n)}\right|^{2}=\mid \int_{0}^{1} d \tau\left(1+e^{i 2 \zeta\left[\vartheta\left(\overline{\mathbf{p}}_{0}-\chi \sin (2 \pi \tau)\right)-\vartheta(\overline{\mathbf{p}}-\chi \sin (2 \pi \tau))\right]}\right)
$$




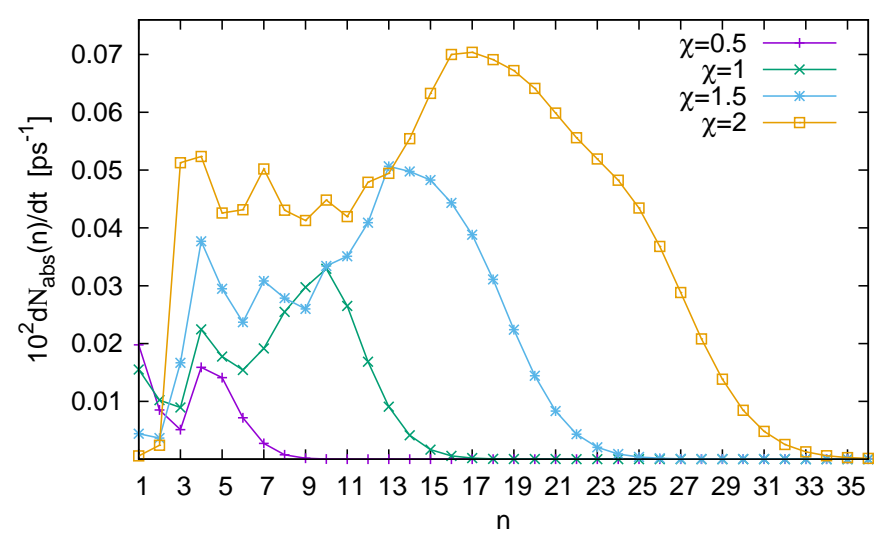

FIG. 4: (Color online) Envelope of partial rate $d N_{a b s}(n) / d t$ of inverse bremsstrahlung absorption vs the mean number of absorbed photons for photon energy $\varepsilon=0.001 \mathrm{eV}$.

$$
\begin{aligned}
\times \exp \{ & i 2 \pi n \tau-\pi i \int_{0}^{\tau}\left[\left(\left(\bar{p}_{x}^{\prime}-\chi \sin \left(2 \pi \tau^{\prime}\right)\right)^{2}+\bar{p}_{y}^{\prime 2}-2 \overline{\mathcal{E}}^{\prime}\right)\right. \\
& \left.\left.-\left(\left(\bar{p}_{x}-\chi \sin \left(2 \pi \tau^{\prime}\right)\right)^{2}+\bar{p}_{y}^{2}-2 \overline{\mathcal{E}}\right)\right] d \tau^{\prime}\right\}\left.\right|^{2}
\end{aligned}
$$

In Eq. (48) the dimensionless momentum, energy, screening vector, time are defined as follows:

$$
\begin{aligned}
& \bar{p}_{x, y}=\frac{p_{x, y}}{\sqrt{m_{*} \hbar \omega}}, \overline{\mathcal{E}}=\frac{\mathcal{E}}{\hbar \omega}, \\
& \overline{\hbar q}_{s}=\frac{\hbar q_{s}}{\sqrt{m_{*} \hbar \omega}}, d \tau=\frac{d t}{T} .
\end{aligned}
$$

For numerical analysis of SB cross-sections in BG the integration in Eq. (48) over scattering angles $d \theta_{\mathbf{p}}, d \theta_{\mathbf{p}^{\prime}}$, will be done by numerical calculations by the fourth-order Runge-Kutta method. We assume Fermi energy $\varepsilon_{F} \simeq \mu=20 \hbar \omega$ $\left(\varepsilon_{F} \gg \hbar \omega\right)$, electrons temperature $T_{e}=0.1 \varepsilon_{F}$, coherent EM linearly polarized radiation field with frequency in terahertz or, close to it, near-infrared $(1.24-124 \mathrm{meV})$ for all calculations. As was mentioned in Sec. II the band dispersion is quadratic and only the lowest subband is occupied [10, 11, 14], and the RPA theory apply in the density domain $10^{10} \mathrm{~cm}^{-2}<n<5 \times 10^{12} \mathrm{~cm}^{-2}$. In particular case for a homogeneous carrier density $n=10^{11} \mathrm{~cm}^{-2}$ the dimensionless effective screening vector $q_{s}$ defined by the formula [60]:

$$
q_{s}=\frac{54.8}{\sqrt{n \times 10^{-10} \mathrm{~cm}^{2}}}
$$

The dielectric environment constant is taken to be $\kappa=2.5$ for an impurity strength in the presence of the $\mathrm{SiO}_{2}$ substrate [62]. To reveal the peculiarities which can be associated with the chiral nature of BG and its parabolic dispersion, the comparison with the SG case has been made.

The envelope of the partial rate of inverse-bremsstrahlung absorption in doped BG is shown for different wave intensities in Fig. 1-4 for photon energies $\varepsilon \equiv \hbar \omega=0.01 \mathrm{eV}\left(\lambda=1.24 \times 10^{-2} \mathrm{~cm}\right), \varepsilon=0.005 \mathrm{eV}\left(\lambda=2.48 \times 10^{-2}\right.$ $\mathrm{cm}), \varepsilon=0.0025 \mathrm{eV}\left(\lambda=0.5 \times 10^{-2} \mathrm{~cm}\right)$, and $\varepsilon=0.001 \mathrm{eV}(\lambda=0.124 \mathrm{~cm})$, respectively. As was expected the results substantially depend on coherent radiation frequency and intensity: $I_{\chi}=\chi^{2} \times 6 \times 10^{10} \mathrm{Wcm}^{-2}(\hbar \omega / \mathrm{eV})^{3}$. As is seen from these figures, the multiphoton effects become essential already at moderate radiation intensities (with intensity parameter $\chi=0.5$ ). With the increase of coherent radiation intensity, the contribution of the multiphoton absorption processes become larger than the one-photon SB. The maximal multiphoton cut-off number is achieved at $\overline{\mathcal{E}}+n \sim 35$ at the intensity parameter $\chi=2$ for the considering frequencies of the pump wave. Cut-off increases at the increase of the intensity at fixed wave frequency in accordance with the parabolic dispersion of the $A B$ stacked BG. In comparison with the doped intrinsic SG [18], where for the same interaction parameters one has a single maximum case, we can note, that the envelopes of the partial rate of inverse bremsstrahlung absorption have many 


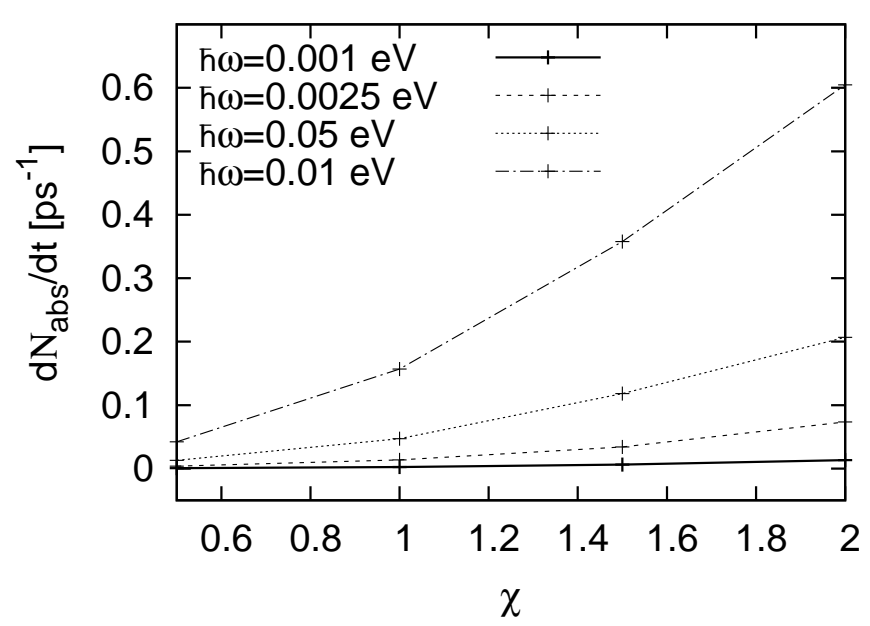

FIG. 5: Total rate of inverse bremsstrahlung in doped graphene vs the dimensionless parameter $\chi$ for the setup of Fig. 1 at various photon energies $\varepsilon$.

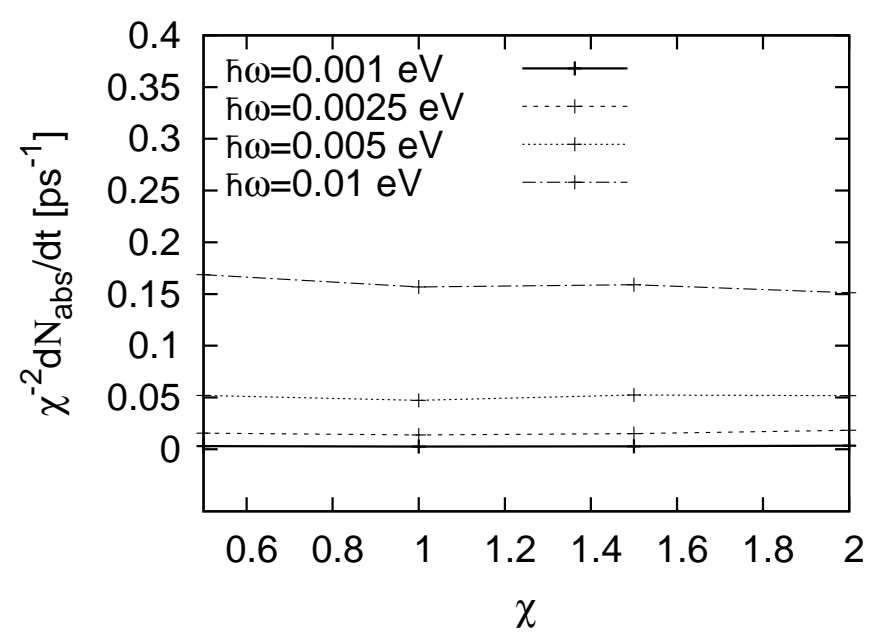

FIG. 6: Total rates of the inverse bremsstrahlung absorption scaled to $\chi^{2}$ vs the parameter $\chi$.

characteristic maxima (minima) in the doped BG. From the comparison of Figs. 1-4 we can conclude that the partial rate of the absorption process in doped BG increases with the increase of the photon energy at the fixed parameter $\chi$ of the pump wave intensity, or with the increase of $\chi$ at the fixed photon energy.

The numerical results allow to demonstrate the dependence of the inverse bremsstrahlung absorption rate on the coherent radiation intensity. So, in Fig. 5 the total SB rate (40) via the mean number of absorbed photons per impurity ion, per unit time in doped graphene versus the parameter $\chi$ for various photon energies is presented. In Fig. 6 we plot the scaled absorption rate $\chi^{-2} d N_{a b s} / d t$ versus the intensity parameter $\chi$. Figure 6 demonstrates, that almost for all considered photon energies the scaled SB rate is constant, which means that even for intense radiation when the multiphoton effects are essential, the absorption rate is proportional to wave intensity. The latter means that with the increase of the wave intensity we approach to a quasiclassical regime of interaction: $\mathcal{E}^{\prime}-\mathcal{E}>>\hbar \omega$ and $\mathcal{E}^{\prime}-\mathcal{E} \sim \chi^{2}$. For terahertz and near-infrared photons (wavelengths from $3 \mu \mathrm{m}$ to $3 \mathrm{~mm}$ ), the multiphoton interaction mode in BG can be achieved at intensities $I_{\chi} \sim 10^{3} \mathrm{Wcm}^{-2}-10^{5} \mathrm{Wcm}^{-2}$, which are available in present [63], [64]. Thus, for these intensities of the strong coherent radiation field, one can manipulate with the dressed charged carriers transport properties of doped BG by the mechanism of multiphoton SB absorption. 


\section{CONCLUSION}

The microscopic quantum theory of multiphoton SB absorption in doped BG ( $A B$ stacked) in the coherent strong EM radiation of arbitrary intensity has been presented. The taken terahertz or near-infrared frequencies and Fermi energies have allowed excluding the chiral particles' interband transitions. We have solved the Liouville-von Neumann equation for the density matrix. The external wave-field has been taken exactly. The charged impurity ions arbitrary electrostatic potential is considered in the Born approximation. These solutions for SB at the linear polarization of EM wave are used for derivation of a relatively simple formula for the multiphoton SB absorption rate. The chiral fermions in doped BG are represented by the grand canonical ensemble. The obtained analytical formulas have been analyzed numerically for screened Coulomb potential. The obtained results have an essentially nonlinear dependence on the increase of the wave intensity. In comparison with the SG case, the new behavior has been demonstrated, which may be associated with its parabolic energy dispersion. In particular, there are many characteristic maxima (minima) of the envelopes of the partial rate of inverse bremsstrahlung absorption in a case of the doped BG. The multiphoton absorption or emission processes play a significant role already at moderate pump wave intensities $I_{\chi} \sim 10^{3} \mathrm{Wcm}^{-2}$. The requirement of high intensity in the terahertz regime does not preclude the use of standard terahertz lasers, which are available [64]. Especially, of interest is the mid-infrared frequencies' range, where Quantum Cascade lasers 65] are readily available and can provide higher powers. So, for such intensities, the multiphoton SB process opens new channels for the wave absorption. It is shown that one can achieve the efficient absorption coefficient by the SB process for pump wave frequencies in considering domain.

\section{Acknowledgments}

The authors are deeply grateful to prof. H. K. Avetissian for permanent discussions and valuable recommendations. This work was supported by the RA MES State Committee of Science.

[1] K. S. Novoselov, A. K. Geim, S. V. Morozov, D. Jiang, Y. Zhang, S. V. Dubonos, I. V. Grigorieva, A. A. Firsov, "Electric field effect in atomically thin carbon films", Science 306(5696), 666-669 (2004), http://dx.doi.org/10.1126/science.1102896

[2] A. K. Geim, "Graphene: Status and prospects", Science 324(5934), 1530-1534 (2009), http://dx.doi.org/10.1126/science.1158877.

[3] M. I. Katsnelson and K. S. Novoselov, "Graphene: new bridge between condensed matter physics and quantum electrodynamics", Solid State Commun. 143(1-2), 3-13 (2007), http:// dx.doi.org/10.1016/j.ssc.2007.02.043.

[4] M. I. Katsnelson, K. S. Novoselov, A. K. Geim, "Chiral tunnelling and the Klein paradox in graphene", Nature Phys. 2, 620-625 (2006), http://dx.doi.org/10.1038/nphys384.

[5] A. H. Castro Neto, F. Guinea, N. M. R. Peres, K. S. Novoselov, A. K. Geim, "The electronic properties of graphene", Rev. Mod. Phys. 81(1), 109-162 (2009), http://dx.doi.org/10.1103/RevModPhys.81.109

[6] K. S. Novoselov, A. K. Geim, S. V. Morozov, D. Jiang, M. I. Katsnelson, I. V. Grigorieva, S. V. Dubonos, and A. A. Firsov, "Two-dimensional gas of massless Dirac fermions in graphene", Nature 438, 197-200 (2005), http://dx.doi.org/10.1038/nature04233.

[7] S. Das Sarma, A. K. Geim, P. Kim, and A. H. MacDonald Recent, "Exploring graphene: research advances", A special issue of Solid State Communications 143(1-2), pp. 1-122 Elsevier, New York, 2007.

[8] E. McCann and V. I. Fal'ko, "Landau-level degeneracy and quantum Hall effect in a graphite bilayer", Phys. Rev. Lett. 96, 086805(1)-086805(4) (2006), https://doi.org/10.1103/PhysRevLett.96.086805.

[9] M. Koshino and T. Ando, "Transport in bilayer graphene: calculations within a self-consistent Born approximation", Phys. Rev. B 73, 245403(1)-245403(8) (2006), https://doi.org/10.1103/PhysRevB.73.245403.

[10] I. Snyman and C. W. J. Beenakker, "Ballistic transmission through a graphene bilayer", Phys. Rev. B 75, 045322(1)045322(6) (2007), https://doi.org/10.1103/PhysRevB.75.045322.

[11] S. Morozov, K. Novoselov, M. Katsnelson, F. Schedin, D. Elias, J. Jaszczak, and A. Geim, "Giant intrinsic carrier mobilities in graphene and its bilayer", Phys. Rev. Lett. 100, 016602(1)-016602(4) (2008), https://doi.org/10.1103/PhysRevLett.100.016602.

[12] K. Novoselov, E. McCann, S. V. Morozov, V. I. Fal'ko, M. I. Katsnelson, U. Zeitler, D. Jiang, F. Schedin, and A. K. Geim, "Unconventional quantum Hall effect and Berry's phase of 2pi in bilayer graphene", Nature Phys. 2, 177-180 (2006), https://doi.org/10.1038/nphys245.

[13] J. Oostinga, H. B. Heersche, X. Liu, A. F. Morpurgo, L. M. Vandersypen, "Gate-induced insulating state in bilayer graphene devices", Nature Mater. 7, 151-157 (2008), http://dx.doi.org/10.1038/nmat2082.

[14] E. H. Hwang and S. Das Sarma, "Screening, Kohn anomaly, Friedel oscillation, and RKKY interaction in bilayer graphene", Phys. Rev. Lett. 101, 156802(1)-156802(4) (2008), http://dx.doi.org/10.1103/PhysRevLett.101.156802. 
[15] F. Hipolito, A. Taghizadeh, and T. Garm Pedersen, "Nonlinear optical response of doped mono- and bilayer graphene: Length gauge tight-binding model", Phys. Rev. B. 98, 205420(1)-205420(11) (2018), http://dx.doi.org/10.1103/PhysRevB.98.205420.

[16] H. K. Avetissian, "Relativistic Nonlinear Electrodynamics: The QED Vacuum and Matter in Super-Strong Radiation Fields", monograph: 978-3-319-26384-7, Springer, New York, 2016.

[17] A. K. Avetissian, A.G. Ghazaryan, K. V. Sedrakian, and B. R. Avchyan, "Induced nonlinear cross sections of conductive electrons scattering on the charged impurities in doped graphene", J. Nanophoton. 11, 036004(1)-036004(11) (2017), https://doi.org/10.1117/1.JNP.11.036004.

[18] A. K. Avetissian, A.G. Ghazaryan, K. V. Sedrakian, and B. R. Avchyan, "Microscopic nonlinear quantum theory of absorption of strong EM radiation in doped graphene", J. Nanophoton. 12, 016006(1)-016006(12) (2018), https://doi.org/10.1117/1.JNP.12.016006.

[19] H. K. Avetissian, G. F. Mkrtchian, "Coherent nonlinear optical response of graphene in the quantum Hall regime", Phys. Rev. B 94(4), 045419(1)-045419(7) (2016), http://dx.doi.org/10.1103/PhysRevB.94.045419.

[20] H. K. Avetissian, A. G. Ghazaryan, H. H. Matevosyan, G. F. Mkrtchian, "Microscopic nonlinear relativistic quantum theory of absorption of powerful x-ray radiation in plasma", Phys. Rev. E 92, 043103(1)-043103(8) (2015), http://dx.doi.org/10.1103/PhysRevE.92.043103

[21] H. K. Avetissian, G. F. Mkrtchian, K. G. Batrakov, S. A. Maksimenko, A. Hoffmann, "Nonlinear theory of graphene interaction with strong laser radiation beyond the Dirac cone approximation: Coherent control of quantum states in nano-optics", Phys. Rev. B 88, 245411(1)-245411(7) (2013), http://dx.doi.org/10.1103/PhysRevB.88.245411.

[22] H. K. Avetissian, G. F. Mkrtchian, K. G. Batrakov, S. A. Maksimenko, A. Hoffmann, "Multiphoton resonant excitations and high-harmonic generation in bilayer grapheme", Phys. Rev. B. 88, 165411(1)-165411(9) (2013), http://dx.doi.org/10.1103/PhysRevB.88.165411.

[23] H. K. Avetissian, A. K. Avetissian, G. F. Mkrtchian, Kh. V. Sedrakian, "Multiphoton resonant excitation of FermiDirac sea in graphene at the interaction with strong laser fields", J. Nanophoton. 6, 061702(1)-061702(17) (2012), http://dx.doi.org/10.1117/1.JNP.6.061702

[24] H. K. Avetissian, A. G. Ghazaryan, G. F. Mkrtchian, Kh. V. Sedrakian, "High harmonic generation in Landauquantized graphene subjected to a strong electromagnetic radiation", J. Nanophoton. 11, 016004(1)-016004(9) (2017), http://dx.doi.org/10.1117/1.JNP.11.016004

[25] H. K. Avetissian, A. G. Ghazaryan, G. F. Mkrtchian, "Relativistic theory of inverse-bremsstrahlung absorption of ultrastrong laser radiation in plasma", J. Phys. B 46, 205701(1)-205701(9) (2013), http://dx.doi.org/10.1088/0953-4075/46/20/205701

[26] H. K. Avetissian, A. K. Avetissian, G. F. Mkrtchian, Kh. V. Sedrakian, "Creation of particle-hole superposition states in graphene at multiphoton resonant excitation by laser radiation", Phys. Rev. B 85(11), 115443(1)-115443(10) (2012), http://dx.doi.org/10.1103/PhysRevB.85.115443.

[27] H. K. Avetissian, A. K. Avetissian, A. G. Ghazaryan, Kh. V. Sedrakian, and G. F. Mkrtchian, "High-order harmonic generation in gapped bilayer graphene", preprint arXiv:1905.08016, https://arxiv.org/abs/1905.08016.

[28] A. K. Avetissian, A. G. Ghazaryan, Kh. V. Sedrakian, "Third harmonic generation in gapped bilayer graphene", preprint arXiv:1905.08189, https://arxiv.org/abs/1905.08189.

[29] K. Xu, S. Liu, W. Sun, Zh. Ma, Z. Li, Qi Yu, G. Li, "Design and fabrication of a monolithic optoelectronic integrated Si CMOS LED based on hot-carrier effect", IEEE Journal of Selected Topics in Quantum Electronics 22, Issue: 6, Nov.-Dec. (2016), http://dx.doi.org/10.1109/JSTQE.2016.2517980

[30] R. R. Nair, P. Blake, A. N. Grigorenko, K. S. Novoselov, T. J. Booth, T. Stauber, N. M. R. Peres, A. K. Geim, "Fine structure constant defines visual transparency of graphene", Science 320, 1308 (2008), http://dx.doi.org/10.1126/science.1156965

[31] J. Liang, J. Lianga, Y. Wanga, Y. Huanga,Y. Maa, Z. Liua, J. Caib, Ch. Zhangb, H. Gaob, Y. Chena, "Electromagnetic interference shielding of graphene/epoxy composites", Carbon 47, 922-925 (2009), http://dx.doi.org/10.1016/j.carbon.2008.12.038

[32] T. Low, P. Avouris, "Graphene plasmonics for terahertz to mid-infrared applications", ACS Nano 8, 1086-1102 (2014), http://dx.doi.org/10.1021/nn406627u

[33] F. V. Bunkin, A. E. Kazakov, M. V. Fedorov, "Interaction of intense optical radiation with free electrons (nonrelativistic case)", Sov. Phys.-Usp. 15, 416-435 (1973), http://dx.doi.org/10.1070/PU1973v015n04ABEH004990.

[34] T. Ando, "Screening effect and impurity scattering in monolayer graphene", J. Phys. Soc. Jpn. 75, 074716(1)-074716(7) (2006), http://dx.doi.org/10.1143/JPSJ.75.074716

[35] J.-H. Chen, C. Jang, S. Adam, M. S. Fuhrer, E. D. Williams, D.M. Ishigami, "Charged-impurity scattering in graphene", Nature Physics 4, 377-381 (2008), http://dx.doi.org/10.1038/nphys935.

[36] K. Nomura, A. H. MacDonald, "Quantum Hall ferromagnetism in graphene", Phys. Rev. Lett. 96, 256602(1)-256602(4) (2006), http://dx.doi.org/10.1103/PhysRevLett.96.256602

[37] E. H. Hwang, S. Adam, S. Das Sarma, "Carrier transport in two-dimensional graphene layers", Phys. Rev. Lett. 98, 186806(1)-186806(4) (2007), http://dx.doi.org/10.1103/PhysRevLett.98.186806

[38] D. S. Novikov, "Elastic scattering theory and transport in graphene", Phys. Rev. B 76, 245435(1)-245435(17) (2007), http://dx.doi.org/10.1103/PhysRevB.76.245435.

[39] P. Boross, A. Palyi, "Valley relaxation in graphene due to charged impurities", Phys. Rev. B 92, 035420(1)-035420(13) (2015), http://dx.doi.org/10.1103/PhysRevB.92.035420.

[40] Y.-W. Tan, Y. Zhang, K. Bolotin, Y. Zhao, S. Adam, E.H. Hwang, S. Das Sarma, H. L. Stormer, P. Kim, "Mea- 
surement of scattering rate and minimum conductivity in graphene", Phys. Rev. Lett. 99, 246803(1)-246803(4) (2007), http://dx.doi.org/10.1103/PhysRevLett.99.246803.

[41] M. I. Katsnelson, "Nonlinear screening of charge impurities in graphene", Phys. Rev. B. 74, 201401(1)-201401(3) (2006), http://dx.doi.org/10.1103/PhysRevB.74.201401.

[42] K. Xu, L. W. Snyman, H. Aharoni, "Si light-emitting device in integrated photonic CMOS ICs", Optical Materials 69, 274-282 (2017), http://dx.doi.org/10.1016/j.optmat.2017.03.055

[43] D. P. DiVincenzo, E. J. Mele, "Self-consistent effective-mass theory for intralayer screening in graphite intercalation compounds", Phys. Rev. B 29, 1685-1694 (1984), http://dx.doi.org/10.1103/PhysRevB.29.1685.

[44] N. H. Shon and T. Ando, "Quantum transport in two-dimensional graphite system", J. Phys. Soc. Jpn. 67, 2421-2429 (1998), http://dx.doi.org/10.1143/JPSJ.67.2421

[45] H. Suzuura, T. Ando, "Crossover from symplectic to orthogonal class in a two-dimensional honeycomb lattice", Phys. Rev. Lett. 89, 266603(1)-266603(4) (2002), http://dx.doi.org/10.1103/PhysRevLett.89.266603

[46] N. M. R. Peres, F. Guinea, A. H. Castro Neto, "Electronic properties of disordered two-dimensional carbon", Phys. Rev. B 73, 125411(1)-125411(23) (2006), http://dx.doi.org/10.1103/PhysRevB.73.125411

[47] S. Sun, J.-L. Zhu, "Impurity spectra of graphene under electric and magnetic fields", Phys. Rev. B 89, 155403(1)-155403(10) (2014), http://dx.doi.org/10.1103/PhysRevB.89.155403.

[48] S. Adam and S. Das Sarma, "Boltzmann transport and residual conductivity in bilayer graphene", Phys. Rev. B 77, 115436(1)-115436(6) (2008), https://doi.org/10.1103/PhysRevB.77.115436.

[49] M. I. Katsnelson, "Scattering of charge carriers by point defects in bilayer graphene", Phys. Rev. B 76, 073411(1)-073411(3) (2007), https://doi.org/10.1103/PhysRevB.76.073411.

[50] M. Monteverde, C. Ojeda-Aristizabal, R. Weil, K. Bennaceur, M. Ferrier, S. Gueron, C. Glattli, H. Bouchiat, J. N. Fuchs, D.Maslov, "Transport and elastic scattering times as probes of the nature of impurity scattering in single and bilayer graphene", Phys. Rev. Lett. 104, 126801(1)-126801(4) (2010), http://dx.doi.org/10.1103/PhysRevLett.104.126801

[51] W. Zhang and L.-J. Li, "The screening of charged impurities in bilayer graphene", New Journal of Physics 12, 103037(1)103037(6) (2010), http://dx.doi.org/10.1088/1367-2630/12/10/103037

[52] D. K. Patel, A. C. Sharma, "Electron-impurity scattering rate in doped bilayer graphene", AIP Conference Proceedings 1447, 973-976 (2012), https://doi.org/10.1063/1.4710329.

[53] Sh. Xiao, J.-H. Chen, Sh. Adam, E. D. Williams, and M. S. Fuhrer, "Charged impurity scattering in bilayer graphene", Phys. Rev. B 82, 041406(1)-041406(4) (2010), https://doi.org/10.1103/PhysRevB.82.041406.

[54] A. G. Ghazaryan, Kh. V. Sedrakian, "Multiphoton cross sections of conductive electrons stimulated bremsstrahlung in doped bilayer graphene", preprint arXiv:1907.04692, https://arxiv.org/abs/1907.04692.

[55] M. M. Denisov, M. V. Fedorov, "Bremsstrahlung effect on relativistic electrons in a strong radiation field", Sov. Phys. JETP 26, 779-783 (1968), http:// jetp.ac.ru/cgi-bin/dn/e_022_04_0844.pdf.

[56] T. R. Hovhannisyan, A. G. Markossian, G. F. Mkrtchian, "On the theory of the relativistic cross-sections for stimulated bremsstrahlung on an arbitrary electrostatic potential in the strong electromagnetic field", Eur. Phys. J. D 20, 17-25 (2002), http://dx.doi.org/10.1140/epjd/e2002-00110-7.

[57] J. Nilsson, A. H. Castro Neto, F. Guinea, and N. M. R. Peres, "Electronic properties of graphene multilayers", Phys. Rev. Lett. 97, 266801(1)-266801(4) (2006), https://doi.org/10.1103/PhysRevLett.97.266801.

[58] J. Nilsson, A. H. Castro Neto, F. Guinea, and N. M. R. Peres, "Electronic properties of bilayer and multilayer graphene", Phys. Rev. B 78, 045405(1)-045405(7) (2008), https://doi.org/10.1103/PhysRevB.78.045405.

[59] T. Ando, A. B. Fowler, F. Stern, "Electronic properties of two-dimensional systems", Rev. Mod. Phys. 54, 437-672 (1982), http://dx.doi.org/10.1103/RevModPhys.54.437

[60] S. D. Sarma, Sh. Adam, E. H. Hwang, and E. Rossi, "Electronic transport in two-dimensional graphene", Rev. Mod. Phys. 83, 407-415 (2011), http://dx.doi.org/10.1103/RevModPhys.83.407

[61] E. H. Hwang and S. Das Sarma, "Dielectric function, screening, and plasmons in two-dimensional graphene", Phys. Rev. B. 75, 205418(1)-205418(6) (2007), https://doi.org/10.1103/PhysRevB.75.205418.

[62] S. Das Sarma, E. H. Hwang, and E. Rossi, "Theory of carrier transport in bilayer graphene", Phys. Rev. B 81, 161407R(1)161407(4)R (2010), http://dx.doi.org/10.1103/PhysRevB.81.161407.

[63] Stanford Linear Accelerator Center (SLAC): https://www6.slac.stanford.edu.

[64] X. Ch. Zhang, A. Shkurinov and Y. Zhang, "Extreme terahertz science", Nature Photonics 11, 16-18 (2017), https://doi.org/10.1038/nphoton.2016.249.

[65] Quantum Cascade Lasers: https://www.teamwavelength.com/quantum-cascade-laser-basics/. 